\title{
EL PROBLEMA DE UNA SUPERACIÓN TÉCNICA EN EL CUERPO DANZANTE
}

\author{
The problem of a technical improvement in a dancing body \\ Paulina Morales Guzmán \\ Universidad Diego Portales, Santiago, Chile \\ plmorales@uc.cl
}

\section{Resumen}

En el presente trabajo se busca problematizar el vínculo entre el cuerpo danzante y la técnica, entendida como la normatividad que guía los movimientos, a la luz del problema general de la expresión. A partir de las propuestas desarrolladas por Maurice Merleau-Ponty en Lo visible y lo invisible y Jean-Luc Nancy en Corpus, se pretende analizar la relación del cuerpo con su entorno espacial con vistas a afirmar un cuerpo que exceda al cuerpo-cosa y con ello hacer crítica a la idea de una mejora o 'superación técnica' del cuerpo en su ejecución danzante.

Palabras clave: danza, cuerpo, técnica, espacio, quiasmo.

\begin{abstract}
The present work seeks to problematize the link between the dancing body and the technique, understood as the normative that guides the movements, given the general problem of expression. From the proposals developed by Maurice Merleau-Ponty in The visible and the invisible and JeanLuc Nancy in Corpus, it is intended to analyze the relationship of the body with its spatial environment with the purpose of affirming a body that exceeds the body-thing and thus to make criticism of the idea of an enhance or 'technical improvement' of the body in its dancing execution.
\end{abstract}

Keywords: dance, body, technic, space, chiasm.

Fecha de Recepción: 01/07/2019 - Fecha de Aceptación: 26/11/2019 


\section{Introducción}

La danza es el espacio en que se evidencia lo problemático que es pensar al individuo como tal, es decir, monádico, determinado $\mathrm{y}$, principalmente, delimitado, en la medida en que el bailarín se dispone siempre en relación con un entorno, jugando con las formas del espacio y anulando, con ello, su individualidad: el bailarín siempre está en relación con lo otro. El cuerpo en la danza se distingue de otros cuerpos por presentarse fluido y en un movimiento que parece hacerlo inmune a ciertas leyes de la física: Paul Valéry lo compara con una llama (1990 180), porque ella se observa como el fenómeno de "consumo interno de una energía" (Ibid.), es decir, para Valéry, el cuerpo danzante es una intersección de fuerzas del mundo como movimiento visible de las energías del espacio. La existencia corporal del bailarín -esto es, la relación que hay entre este cuerpo y un espacio circundante- es el fenómeno que invita a pensar el cuerpo ya no solo en un vínculo con el espacio, sino en una coincidencia con él, en la medida en que expresándose no es distinguible del movimiento y la desenvoltura que el cuerpo deviene.

Toda danza, a pesar de la variedad que el mismo concepto alberga, se asocia a una técnica respectiva, esta es, un catálogo de movimientos precisos y determinados sobre los que se basaría el cuerpo en expresión. Dicha técnica es aquella que demarca la diferencia entre estilos y formas de danzas; es, en suma, aquella que las hace distintivas con respecto a otras danzas y otras formas de expresión corporal. Sin embargo, suponer que el cuerpo basa sus movimientos en aquel catálogo resulta problemático pues, esto supondría que la danza no consiste en otra cosa que en la reproducción de signos corporales y que el cuerpo, en esta empresa, existe como un mero instrumento, cosa material utilizada por un sujeto que no expresa. Y es que la expresión implica una emergencia de movimientos nuevos, la re-articulación del espacio en que el cuerpo se desenvuelve. Por lo tanto, si se busca afirmar al cuerpo danzante como cuerpo que expresa, es necesario cuestionar la acepción normativa que tiene la técnica, con lo cual se discutirá aquel rol de 'base'. Por consiguiente, se propone pensar un cuerpo danzante que excede la mera normatividad de la técnica.

La noción 'técnica', por lo general, suele ser empleada como si se tratase de una res disponible para el uso del cuerpo, ya que usualmente se habla de 'manejo técnico', de 'buen o mal dominio de la técnica' y, por sobre todo, de 'superación técnica' de un cuerpo, empleando la 'superación' como la evolución o mejora del cuerpo en su manejo práctico de aquella res técnica. No obstante, al suponer que existe algo así como una 'superación técnica' del cuerpo, además de suponer que hay algo así como una res técnica, se admite también la reificación y delimitación del cuerpo que danza, en suma, técnica y cuerpo como dos totalidades que en el 
movimiento deben enfrentarse. Con ello, el cuerpo que se supera técnicamente significaría uno que se mueve ad hoc a la normatividad y que, en virtud de esa adecuación, aumenta sus capacidades motrices. Pero el cuerpo que danza, en tanto que llama, fuego, no es dominado, sino expresivo.

En este trabajo se busca problematizar la idea de una 'superación técnica', entendida como la instancia de un cuerpo que evoluciona en sus capacidades motrices en virtud del dominio de una técnica. Para ello, se propondrá la comprensión del cuerpo danzante como aquel que va más allá de lo que se entiende como el cuerpo meramente anatómico del bailarín, esto es, cuerpo en tanto cosa. Es posible problematizar la 'superación' técnica del cuerpo en la medida en que se ahonde en la relación entre cuerpo y técnica en la danza; así, nuestra propuesta del cuerpo danzante permitiría entender la técnica ya no como una normatividad que entorpece la expresión del cuerpo desde una intervención externa al movimiento, sino más bien como aquello que siempre está incorporado en el cuerpo que danza, incorporación que la anula como normatividad. Para pensar el cuerpo y su quehacer técnico en la danza, nos centraremos en las propuestas que Maurice Merleau-Ponty trata en su escrito póstumo Lo visible y lo invisible. En este escrito el autor desarrolla una noción de cuerpo que problematiza la propiedad de este a una consciencia o, incluso, a una sensibilidad localizada. Luego se vinculará esta propuesta con la noción del cuerpo presente en Corpus de Jean-Luc Nancy, a partir de la cual se ahondará en el exceso del cuerpo, cuerpo que no admite lugar a una res técnica. A partir de ambas propuestas se buscará hacer una crítica a la idea de una mejora o 'superación técnica' del cuerpo a partir de su quehacer danzante.

\section{1.}

En una comprensión científica, o si se quiere, anatómica del cuerpo humano, todo bailarín requiere desarrollar ( $\mathrm{y}$, para ello, entrenar) ciertas capacidades de su cuerpo, para así poder llevar a cabo la ejecución de los movimientos a plenitud, sin la intervención de las incapacidades de este cuerpo-cosa -como la tensión de los músculos, el desequilibrio, etc. Por ello, para el cuerpo de atletas y bailarines sería menester una preparación técnica en vista de los movimientos que ejecutará, pues, para este cuerpo-cosa es necesario que las capacidades se incrementen con entrenamiento, en vistas de un desarrollo o, si se quiere, una evolución de capacidades físicas. Sin embargo, el cuerpo no acaba de definirse en su acepción anatómica u objetual: el cuerpo en movimiento no se debe a su solo mecanismo muscular, pues la expresión emerge justamente porque el cuerpo no es una mera cosa. Y dado que la superación técnica del cuerpo se piensa en base al cuerpo-cosa, un replanteamiento del cuerpo en movimiento repercute en su relación con la 
técnica -si es que la hay. Entonces, cabe cuestionarse si es posible mantener la idea de una mejora o superación técnica del cuerpo pensándola desde su vínculo experiencial con el mundo.

Merleau-Ponty, en su obra póstuma Lo visible y lo invisible, a partir del fenómeno del 'quiasmo' o entrelazo invita a pensar en un cuerpo que ya no está totalmente inmerso en la carne del mundo -inmersión que el autor desarrolla en sus primeras obras a partir de su noción del 'gesto'. El quiasmo introduce en la relación del cuerpo con el mundo una distancia: "El quiasmo, reversibilidad, es la idea de que toda percepción está acompañada de una contra-percepción [...], ya no se sabe quién habla ni quién escucha" (Merleau-Ponty 2010 233). La pérdida del 'quién' es el aspecto que se buscará ahondar a continuación en torno a la danza, enfatizando en que el cuerpo danzante no se limita a ser aquel cuerpo-cosa movido por un 'quién' determinado. La ambigüedad que emerge con el quiasmo nos dice que el cuerpo pensado desde esta contra-percepción cambia la forma en que se entiende su relación con el mundo. Por lo tanto, si se busca ahondar en la relación entre el cuerpo danzante y la técnica, es necesario precisar cómo entender la relación entre el cuerpo y el mundo, ya que dicha relación es el lugar de la danza y su técnica.

El quiasmo es introducido como fenómeno que emerge en la experiencia del tacto. Sin embargo, antes de presentarnos el entrelazo, Merleau-Ponty desarrolla la manera tradicional de entender las sensaciones. Esto, para enfatizar la necesidad que existe en aquella comprensión de dos elementos principales que responden a la lógica de sujeto/objeto. Siguiendo este mismo movimiento, se desarrollará el cuerpo desde el quiasmo y su relación con la técnica aboliendo la necesidad de pensarlos a partir de la dualidad sujeto/objeto.

Entonces, el autor inicia su examen acerca de la dualidad sujeto/objeto analizando la visibilidad en tanto que fenómeno sensible del cuerpo: "Lo visible alrededor de nosotros parece descansar en sí mismo. Es como si nuestra visión se formara en su centro o como si hubiera entre él y nosotros una intimidad tan estrecha como la del mar y la playa" (Merleau-Ponty 2010 119). Merleau-Ponty resalta de este ejemplo la dependencia que hay entre los elementos vidente y visible para sostenerse como tal. Continúa inmediatamente después, agregando que "no es posible que nos fundamos en él [lo visible], ni que él pase a nosotros, porque entonces la visión de desvanecería [...]" (Ibid., inserción mía). Entonces, tradicionalmente, para afirmar la visión en tanto tal, sería necesaria una dependencia de dos partes, un visible y un vidente, los cuales deben entenderse como diferentes e independientes uno de otro de manera que se mantenga la relación que sería la visión.

Se trata de una visión que parece estar tendiente hacia un centro que es lo visible, de una visión que es yoica, de un 'yo' cuya interioridad es condición de 
posibilidad para su encuentro con un mundo al que se tiende. Esta visibilidad presupone un aislamiento del 'yo' frente a un mundo-cosa; $y$, frente a un mundocosa el 'yo' necesariamente se afirma como sujeto a un cuerpo-cosa. $\mathrm{Y}$ entendiendo al sujeto de esta forma, el cuerpo del bailarín se limita a ser res instrumental del 'yo', y cuya relación con el mundo es indirecta, mediada por este 'yo' que tematiza tanto a su cuerpo-cosa como al mundo-cosa para establecer una interacción en la forma de una danza. En dicha comprensión del cuerpo, la técnica viene a ser otra cosa tematizada por el 'yo', cosa del mundo que se adecuaría al cuerpo. No obstante, un cuerpo tal pierde su vínculo con el mundo al no ser pensado en su experiencia in situ de sus movimientos y de su desenvoltura en el espacio. Por ello, esta forma de plantear a las sensaciones se vuelve problemática para MerleauPonty, ya que se presupone un límite entre cuerpo y mundo al ignorar las sensaciones como vínculo.

Así, Merleau-Ponty ofrece una nueva manera de plantear la visión que considere el cuerpo del mundo sin obviar su característica sintiente. Dicha carnalidad del cuerpo sintiente supone una relación que no implica un enfrentamiento entre yo y otro (vidente y visible). Para introducir este planteamiento, recurre al fenómeno del tacto, tomando el ejemplo husserliano de la mano tocante y tocada ( $c f$. Husserl 184), en la que emerge el fenómeno que busca introducirnos: el quiasmo. En el tacto se evidencia un atisbo de ajenidad indeterminable en aquello que la lógica yoica reconoce como mi cuerpo.

Es menester que entre la exploración [de mi mano] y lo que ella me enseñará, entre mis movimientos y lo que yo toco, exista alguna relación de principio, algún parentesco según el cual ellos [son] [...] la iniciación y la apertura a un mundo táctil. Esto sólo puede suceder si, al mismo tiempo que sentida desde adentro, mi mano es también accesible desde afuera: [...] (2010 121-122).

El primer aspecto que Merleau-Ponty introduce es la necesidad de una "apertura al mundo táctil", esto es, apertura como una instancia gestual en la que el cuerpo sintiente se imbrica en el mundo, de tal forma que ya no hay mundo como objetividad frente a un cuerpo subjetivo, sino que ambos, cuerpo y mundo, pierden todo estatuto en sí para volverse uno: la carne de mundo. Entonces, la apertura al mundo táctil mencionada es la existencia del cuerpo inmerso gestualmente en un mundo que también es carne - reconocido como tal en función de su indistinción de lo sintiente en el gesto. Luego, se indica un elemento que va más allá de la absoluta inmersión en la carne de mundo: es la necesidad de un cuerpo que sea sentido tanto en un dentro como en un afuera. Esta forma de pensar el cuerpo introduce la necesidad de un afuera que no implique, por ello, reificación, es decir, la vuelta a un cuerpo cerrado frente a lo visible del mundo. 
A través del ejemplo husserliano de una mano que toca a otra, MerleauPonty resalta la instancia en la que el cuerpo se experimenta propio, dadas las sensaciones localizadas en la mano tocada, pero que, a su vez, se experimenta como objeto del mundo: "un verdadero tacto del tocar, cuando mi mano derecha toca a mi mano izquierda palpando las cosas, por el cual el "sujeto que toca" pasa al rango de tocado, desciende a las cosas, de manera que el tacto se hace desde el medio del mundo y como en ellas" (2010 122). En principio, el autor sitúa al sujeto en un rango vacilante, proponiéndolo entre comillas como 'sujeto que toca', y esto es justamente por lo que busca afirmar: llega un punto en que este ser-tocante se ve excedido por un ser-tocado. Este 'punto' es una instancia particular del cuerpo en que se encuentra inmerso en un mundo abierto a sí (táctilmente, en el ejemplo), pero que, a su vez, se le resiste, manteniendo una distancia que lo muestra como cuerpo (como mano-aquí). Con este nuevo elemento, el cuerpo que danza no solo puede pensarse meramente fusionado con el mundo que lo recoge en su danza, ya que es necesario agregar una distancia con este que, a su vez, no lo reduzca a la corporalidad anatómica dominada por la técnica.

Luego, en el fragmento citado, Merleau-Ponty afirma que "el tacto se hace desde el medio del mundo" y como en las cosas mismas. Un 'medio' del mundo tiene sentido bajo la imagen de un mundo allá, lejano, y un mundo acá, el inmediatamente sensible, el de la chair del ser-tocante. Entonces nos está ilustrando un punto del mundo de lo sensible que es ambiguo, que está entre la reificación del ser-tocado y la ubicuidad del ser-tocante. Y esta ambigüedad es el espacio que hace al cuerpo exceso de sí - se excede en su ser-chair, tocante e inmersa.

Dicho exceso tiene que ver con un cuerpo que, en un cierto grado de inmersión con el mundo - esto es, como anulación de un cuerpo yoico para ser uno con todo lo otro-, se encuentra a su vez presente como cuerpo interpelado por el mundo, en la medida en que siendo tocado hay una mano que se siente y se afirma tocante, esto es, intencionada al mundo. De este mismo modo, cuando ya no hay un cuerpo delimitado por un cierto rango de capacidades, la relación de este con la técnica ya no es de un intercambio o intencionalidad entre dos elementos independientes entre sí; uno frente al otro como vidente y visible. Cuerpo y técnica son, en la danza, elementos tan intercambiables como lo es la mano tocante-tocada.

Si se retoma la primera cita expuesta en este texto referente al quiasmo, en la que Merleau-Ponty indica que con el quiasmo "ya no se sabe quién habla ni quién escucha" (2010 233), ahora es posible constatar que este fenómeno designa una ruptura en la que se pierde el centro yoico, pero sin suponer por ello una completa unión con el mundo: justamente el conflicto está en un mundo que interpela a una presencia sensible (cuerpo) que, al mismo tiempo, sin un centro determinado desdibuja el límite entre lo interior y lo exterior, esparciéndose a lo 'otro'. Es este límite desdibujado lo que justamente problematiza el hablar de lo interior y lo otro 
como tal, ya que lo 'otro' está reconociéndose como uno mismo en lo sensible, y lo que es propio fenoménicamente es a la vez ajeno y distante.

\section{2.}

Ahora bien, si la carne de mundo invita a pensar en un punto medio entre sujeto y mundo, ¿qué lugar tiene la técnica para un cuerpo que no supone una interioridad previa? Esta ya no puede ser pensada en los mismos términos en que se han introducido anteriormente, pues depende de un cuerpo pensado como totalidad. En efecto, para afirmar la existencia de una superación técnica en la danza es necesaria una medida según la cual se reconozcan estatutos del cuerpo y el avance del mismo en rangos 'mejores' o 'mayores'. Entonces, un cuerpo sujeto a la superación técnica necesita, en primera instancia, estar delimitado, de modo que se le pueda reconocer suplementado por una técnica - en la medida que 'superación' implica adhesión para una mejora, con respecto a un cuerpo-cosa que por sí solo no es suficiente. A su vez, esto implica una segunda instancia, la de un cuerpo que se diferencie del mundo, de manera que se conciba la idea de un cuerpo en necesidad de dominio técnico, es decir, un cuerpo que domina el espacio que usa. Esto implica una última instancia, la de un cuerpo yoico, cuerpo con agencia de dominio y de tematización del espacio. Pero, reducir el cuerpo que danza a dicho cuerpo-cosa lo hace carecer de su dimensión experiencial, la que necesariamente lo vincula con el mundo.

Por lo tanto, el cuerpo en la danza no es una sustancia pasiva a merced de un mundo autómata, sino que el cuerpo incorpora en su existencia una determinación de sus movimientos, una dirección necesaria hacia la cual el cuerpo tiende, tendencia que prescinde de una finalidad fuera del movimiento mismo. Esta 'tendencia' puede ser ilustrada con el caso que Merleau-Ponty analiza sobre el proceso creativo del pintor Matisse:

[E]s cierto que la mano de Matisse vaciló, es por tanto cierto que hubo elección y que el trazo elegido lo fue de manera que observara veinte condiciones diseminadas sobre el cuadro, informuladas, informulables para cualquier otro que no fuera Matisse, puesto que no estaban definidas e impuestas más que por la intención de hacer aquel cuadro que aún no existía (1964 56).

La elección a la que el autor refiere no es una deliberación llevada a cabo por un sujeto planificador. Para Matisse solo hubo una manera de llevar a cabo aquel cuadro, pero esta manera no conducía a una finalidad supuesta en la elección de cada trazo. Por lo mismo, aquella 'elección' mencionada no puede ser sino un movimiento determinado por la instancia de su creación, pero una determinación que no es causa de un fin -en la medida que no hay fin presupuesto, sino solo 
determinaciones en movimiento. De esta misma manera, la técnica en un movimiento de danza no provee al cuerpo yoico de una opción regulativa para su movimiento, sino que esta misma se vuelve parte de su determinación expresiva. En términos yoicos, es como si el cuerpo que danza no viera más opción en su movimiento que aquel que conlleva una cierta técnica, técnica que no se presenta como res con la que un cuerpo-cosa entra en relación: ya no está como aquel catálogo de movimientos, sino que el cuerpo danzante tiene aquellos movimientos incorporados en su quehacer.

Así, la técnica está en el cuerpo como una forma de situarse en el espacio, pero no ejerciendo dominio sobre este, justamente porque ya no existen delimitadas una frente a la otra. Entonces, la técnica, estos movimientos prefigurados, devienen cuerpo en la instancia misma del movimiento, por lo tanto, ya no siendo aquel catálogo de movimientos definidos, sino disolviéndose como técnica y siendo ahora carne. Ahora bien, si la técnica deviene movimiento, ¿cómo se piensa la superación técnica, que consiste en una adhesión de técnicas, o un aumento de capacidades? El mismo uso de nociones cuantitativas dificulta pensar una superación en un cuerpo que ya no tiene una técnica, sino que la incorpora y la hace movimiento.

\section{3.}

En una conferencia llevada a cabo en 2013 en la Universidad San Francisco de Quito, Nancy analiza la exterioridad del cuerpo como exceso de un modo similar a aquella en que Merleau-Ponty se acerca al quiasmo, en la medida en que recurre a fenómenos experienciales cotidianos del cuerpo. Sin embargo, Nancy no plantea su propuesta de cuerpo en base a la experiencia de la misma, sino situándose como observador de una corporalidad que llama a ser cuestionada en su indeterminación. En dicha conferencia analiza la figura de la cicatriz y la peculiaridad en que ella consiste: “La piel se cierra sobre sí misma inscribiéndose como trazo de lo otro, visible en el afuera y atada al adentro. De manera más exacta, es aquí donde afuera y adentro, de hecho, se desempatan y se empatan" (2015 15). La cicatriz ilustra la dificultad que tiene el cuerpo con concretar sus límites ya que ella consiste en un cierre, al ser la sutura de una apertura, una herida. Pero en tanto que cierre ella es distinta de la piel a la cual se adhiere y con la cual se conecta, por ello es descrita por Nancy como "trazo de lo otro", ya que es la marca de la apertura del cuerpo. La cicatriz ilustra la ambigüedad que tiene la misma piel como límite del cuerpo, límite que definiría su adentro y su afuera $-\mathrm{y}$, con ello, su propio y su ajeno.

Así, la misma piel que permite las sensaciones localizadas se expone en su ambigüedad, al ser un medio transmisor, con poros que hacen de este presunto 
límite del cuerpo anatómico más un canal que un sello. Entonces, el cuerpo, que en la experiencia oscila entre adentros y afueras y entre propiedades y ajenidades, se presenta como aquel complejo al que le son propias todas estas propiedades simultáneamente y, por ende, ninguna, si es entendida por referencia a su opuesto. $\mathrm{Y}$ al entender este cuerpo con esta ambigüedad, se introduce una nueva: el situarse entre pasividad absoluta y pura actividad. Dado que la actividad implica un cuerpo que se enfrenta a un mundo, afectándolo, puede hablarse de un cuerpo activo solo si este se concibe como una interioridad, que es aquel punto desde el cual emerge toda acción. Por otro lado, cuando el cuerpo está inmerso en lo otro, este se encuentra enteramente afuera, donado e impropio, ante lo cual se trataría de un cuerpo pasivo, que es siempre afectado por la alteridad de la cual ya es parte. Ahora bien, la complejidad que Nancy otorga al cuerpo permite pensarlo como un lugar que, en la misma medida en que está existiendo con lo otro, está a su vez distanciándose del mismo - como una cicatriz que es tanto piel, cuerpo que busca cerrar, como ese ajeno, mundo que se inscribe en lo propio: cicatriz que existe siendo lo propio y lo ajeno, sin ser, a su vez, ninguno como tal. Dicha duplicidad del cuerpo invita a pensar nuevamente en un cuerpo que danzando no se relaciona con una técnica, sino que esta se disuelve en la existencia nodelimitada del cuerpo: la falta de una frontera hace del cuerpo un espacio cuyo movimiento no depende de algo otro, sino solo de sí mismo, como un pliegue en sí mismo.

En su texto Corpus, Nancy afirma que "los cuerpos son lugares de existencia, y no hay existencia sin lugar, sin ahí, sin un «aquí», «he aquí», para el éste. El cuerpo lugar no es ni lleno, ni vacío, no tiene ni fuera, ni dentro, como tampoco tiene partes, totalidad, funciones, o finalidad" (2003 16). El cuerpo como lugar es un situarse, pero situarse de ningún centro al cual pertenecer. Entonces, si el cuerpo-lugar supone un ahí, un aquí para sí, este cuerpo es, ante todo, una existencia que no es independiente o cerrada en sí con respecto a algo otro. Por ello, se nos aclara que esto no implica que el cuerpo sea una totalidad, ya que tampoco supone relación con algo otro en la medida en que no es un en sí, lo cual ya podemos notar con su reflexión en torno a la piel.

Ahora, si bien con el quiasmo surge la necesidad de cuestionar la experiencia como totalidad de un 'adentro' corporal, sigue afirmándose en una experiencia que es ella misma un adentro: la experiencia del exceso, aún cuestionando su ser-adentro, se afirma como una localización del sentido -es decir, aquí mi experiencia que conlleva un quiasmo. En cambio, exceder esta experiencia permite que ya no haya necesidad de pensar el afuera desde una idea de dentro de la experiencia: aquí el cuerpo ya no figura atado a una forma de sentido sino solo allí, dispuesto como espacio. Entonces, el cuerpo como lugar de 
existencia en Nancy radicaliza el exceso que Merleau-Ponty nota. Este no se basa en su experiencia del exceso, sino que excede esta experiencia del exceso.

Con ello, ya no hay relación con el mundo pues, ya no hay mundo, y sin un mundo, la técnica ya no puede ser un fenómeno del mundo siquiera tematizable. Así, con las propuestas de Nancy el cuerpo ya no está sujeto a su sentido en la experiencia, y ya no depende de su experiencia in situ para ser pensado como espacio. Si aplicamos esta concepción del cuerpo a la danza, se desvanece toda oportunidad que tenía la técnica como objeto del mundo para tener una relación con el cuerpo danzante. $\mathrm{Y}$ es que un cuerpo que no es totalidad no puede ser pensado en relación con nada sino expuesto, siendo espacio y no estando en relación con él.

Entonces, el cuerpo como exceso no significa uno que es pura salida de sí, justamente porque ser exceso implica que ya no satisface la dualidad dentro/fuera y propio/ajeno. Asimismo, el cuerpo que danza está en una exposición que no implica un existir separado del mundo y que, por lo tanto, no da lugar a una relación con el mismo, ya que una relación presupone la existencia de una distancia que demarque, al menos, dos elementos a vincular. Ahora bien ¿qué lugar tendría la técnica, como aquel catálogo de movimientos, en una supuesta interacción con este cuerpo? Si consideramos que el cuerpo en Nancy no es totalidad, y que al no ser totalidad no hay un otro con el cual cohabitar o interactuar, entonces no hay lugar ontológico para una técnica que se relacione con el cuerpo, sino que solo hay cuerpo-espacio. Entonces, aquello que a un ojo observador de la danza aparece como un cuerpo danzante en dominio de una técnica, no es otra cosa que un cuerpo en constante repliegue, situándose constantemente en nuevas articulaciones. El cuerpo es una existencia que ya no se distingue de otros o de sí mismos.

Entonces, si extendemos las propuestas de Merleau-Ponty y Nancy a la cuestión del cuerpo danzante, podemos plantear que este no danza en función de su técnica ni del dominio de un espacio dado. En efecto, no hay un cuerpototalidad del cual pueda pensarse que la técnica es un implemento que lo suplementa y, a su vez, no hay telos para el cuerpo que brinde criterio con el cual medir superación o disminución. En cambio, el cuerpo que danza no necesita técnica ni espacio, ya que ambos, técnica y espacio, devienen cuerpo danzante, devienen su movimiento, cesando de existir como elementos tematizados anexos al cuerpo. Así, plantear que en el cuerpo danzante pueda haber una superación de su manejo técnico de los movimientos conlleva reducir el cuerpo danzante a un cuerpo-cosa y, con ello, no habla de un cuerpo en expresión y desenvoltura. 


\section{4.}

Nancy nos plantea la idea de un cuerpo que, en tanto espacio, puede pensárselo siempre deviniendo creación en la danza, siempre replegándose para volverse diferente, sin ser otro cuerpo más que aquel espacio en movimiento. Sin embargo, la imagen del cuerpo danzante como diferente de aquel en su quehacer cotidiano invita nuevamente a pensar en la idea de una preparación técnica del cuerpo, es decir, de un cuerpo danzante técnicamente superior al cuerpo cotidiano. En esta línea, Valéry, en su observación de la danza, afirma que el cuerpo danzante es uno que existe de manera paralela a la vida práctica (1990 174). Y este cuerpo, a su juicio, tiene relación con algo que excede la energía estándar de la vida práctica: “el hombre se dio cuenta de que poseía más vigor, más agilidad, más posibilidades articulares y musculares de las que necesitaba para satisfacer las necesidades de su existencia [...]" (Ibid.). A partir de esta observación, más allá de un paralelismo de este cuerpo al cuerpo cotidiano, pareciera que la danza es un ir más allá, conlleva superar un alcance corporal primario para dar cabida a un modo de existencia diferente. Así, Valéry da cuenta de que hay una amplitud de capacidades, nota que hay un más que se añade al cuerpo cotidiano, añadidura que lo hace existir de un modo completamente diferente a su cotidianeidad.

Entonces, el problema de un cuerpo técnicamente superior (esto es, con dominio de una técnica para sus movimientos) parece mantenerse en la observación de un bailarín. Por ello, es necesario hacer el trabajo de ahondar en aquellas circunstancias que se presentan como casos de superación técnica del cuerpo en la danza. Para llevar a cabo este examen, se recurrirá a un movimiento de la técnica del ballet clásico para replantearlo desde las propuestas en torno al cuerpo llevadas a cabo por Nancy y Merleau-Ponty. Uno de los movimientos más recurrentes en el ballet clásico es el grand jeté (salto con apertura de piernas). Este ejercicio comúnmente requiere de un entrenamiento que permita la apertura paulatina de la musculatura, pues la técnica define a este ejercicio a partir de la formación de un ángulo recto entre ambas piernas (es decir, una apertura de $90^{\circ}$ ) (Desrat 1895 192). Sin embargo, hoy en día la apertura de piernas en diferentes disciplinas supera esta medida. La superación en este ejercicio no solo puede ser pensada desde el aumento de la apertura, sino también cuando se agregan a esta flexibilidad elementos de salto o de equilibrio, entre otros. Esta variación de ejercicios puede ser entendida como 'superación' en la medida en que todas suponen como base a la flexibilidad primera: la apertura en ángulo recto.

No obstante, la 'superación' técnica, entendida como una mejora en las capacidades corporales, supone que, guiándonos del ejemplo antes expuesto, aquella apertura mayor de piernas sería un nuevo elemento técnico que reemplaza el grand jeté inicial - lo supera. Pero, con los planteamientos de Nancy sobre el 
cuerpo, entendemos que el cuerpo no es una totalidad que pueda relacionarse con ninguna técnica, ni con la inicial ni con la nueva. Entonces, lo que se observa como 'superación' técnica, en realidad no es otra cosa que un repliegue del cuerpo, es el cuerpo deviniendo un nuevo cuerpo, que, en lugar de dominar una técnica superior, se expone como un espacio nuevo y diferente del anterior, pero sin salir de sí ni volviéndose un cuerpo otro. En efecto, la apertura en ángulo obtuso o extendido en un grand jeté no conlleva la aplicación de una normatividad técnica en la experiencia misma de la ejecución, sino nuevas articulaciones del cuerpo anatómico en el espacio: todo, deviniendo cuerpo, sin anatomía a entrenar ni espacio dominable. Por lo tanto, lo que se observa como una mejora técnica del cuerpo es realmente una nueva puesta en el movimiento. Lo que se observa como una técnica inicial (del grand jeté en ángulo recto) no es inicial en referencia otra ulterior, sino que es una instancia independiente de todo movimiento del cuerpo. $Y$ es que el cuerpo danzante en cada movimiento supone un nuevo pliegue, un nuevo espacio.

\section{Conclusiones}

Tanto los planteamientos de Nancy como las propuestas sobre la expresión de Merleau-Ponty nos permiten concluir que el cuerpo que danza no se limita al cuerpo anatómico: reducir el cuerpo a lo cósico olvida que este es cuerpo en un mundo, que interactúa en su cotidianeidad y que, por ello, es ante todo experiencia. El cuerpo que danza son también el espacio y la técnica, los que tematizamos como tal fuera de la expresividad, pues, aparecen como cosas si son pensadas de forma estática y fuera del cuerpo. La danza, así, es la instancia en que el cuerpo aparece como este espacio de constante movimiento y creación de sí mismo.

Por lo tanto, cuando planteábamos que la técnica solía ser asumida como base de los movimientos del cuerpo en la danza, ahora podemos concluir que su rol de base se anula, en la medida en que la técnica no puede mantener su estatuto de normatividad en la danza, no pudiendo sino devenir cuerpo. La danza no se trata de un cuerpo que crece o evoluciona, sino más bien en la disolución del cuerpo anatómico como eje del movimiento. Con ello, el cuerpo danzante no cambia con entrenamiento de una técnica normativa, ya que este es siempre cambio, constante movimiento, a pesar de cualquier normatividad técnica que busque regir al cuerpo-cosa.

Entonces, cuando Valéry afirmaba que el cuerpo danzante era diferente al de la vida práctica (cotidiana), y cuando califica su energía como "de calidad superior" (1990 180) estaba hablando del cuerpo anatómico de la bailarina, por lo que aquella energía era la de un cuerpo aún delimitado a una subjetividad que se 
relaciona con una técnica y un mundo. Pero el cuerpo danzante no es superior o inferior a nada, pues este es espacio de constante transformación de sí.

Desde Merleau-Ponty a Nancy se ha llevado a cabo un paso desde un cuerpo-en-el-mundo a un cuerpo-espacio. El cuerpo del quiasmo, expuesto por Merleau-Ponty, es uno que está aún instituyéndose: es un cuerpo que se piensa a sí mismo desde la experiencia sensible de ser más allá de la misma - nótese el exceso del ser-tocante al ser-tocado. El cuerpo del quiasmo es el primer hallazgo para entender al cuerpo en la danza, que es sin centro, sin telos y sin origen. Por ello, el cuerpo-espacio que plantea Nancy completa las nociones del quiasmo en MerleauPonty, en la medida en que ya no se supone al cuerpo como aquello que se enfrenta, relaciona o funde con otro, el mundo. Con Nancy, entonces, puede dejar de pensarse en un cuerpo 'en' la danza para formular la noción de un cuerpo de la danza: no hay elementos del mundo que complementen a un cuerpo experimentante, sino que la técnica y el espacio devienen cuerpo y, con ello, el cuerpo deviene danza.

\section{Bibliografía}

Desrat, Gustave. Dictionnaire de la danse. Historique, théorique, practique et bibliographique. Paris: Librairies-Imprimeries Réunies, 1895.

Husserl, Edmund. Ideas relativas a una fenomenología pura y una filosofía fenomenológica II, trad. A. Zirión Q. México: Fondo de Cultura Económica, 2005

Merleau-Ponty, Maurice. Signos, trad. C. Martínez, \& G. Oliver. Barcelona: Seix Barral, 1964.

Merleau-Ponty, Maurice. Lo visible y lo invisible, trad. E. Consigli, \& B. Capdevielle. Buenos Aires: Nueva Visión, 2010

Nancy, Jean-Luc. Corpus, trad. P. Bulnes. Madrid: Arena Libros, 2003.

Nancy, Jean-Luc. "Piel Esencial". Simposio Cuerpos y Corporalidades, eds. C. Burneo Salazar y A. Ordóñez Charpentier. Quito: Universidad de San Francisco de Quito, 2015. 15-23.

Valéry, Paul. "Filosofía de la Danza". Teoría poética y estética, trad. C. Santos. Madrid: Visor, 1990. 